\title{
Heterogeneity within apparently pure cultures of Escherichia coli freshly isolated from significant bacteriuria
}

\author{
R. FREEMAN, P. R. SISSON and D. BURDESS* \\ Regional Public Health Laboratory, Newcastle General Hospital, Newcastle upon Tyne NE4 6BE and \\ * Microbiology Department, Freeman Hospital, Newcastle upon Tyne NE7 7DN
}

\begin{abstract}
Pyrolysis mass spectrometry (PyMS), a highly discriminatory method for comparison of isolates, was used to assess the homogeneity of colonies taken from apparently pure cultures of Escherichia coli in 10 urine specimens. Ten randomly selected colonies were subcultured from each urine for comparison. For six urines, the set of 10 single-colony isolates proved indistinguishable by PyMS. However, for four urines there was clear heterogeneity. For two urines, one of the isolates was distinct, and the remaining nine indistinguishable. One urine yielded seven indistinguishable isolates, and three further individually distinct isolates. One urine yielded two clusters of indistinguishable isolates, one comprising seven and the other three isolates. Thus, significant heterogeneity (as measured by PyMS) occurs in apparently pure cultures of $E$. coli from urinary tract infection. The nature of this heterogeneity remains to be established, as does the significance, if any, of this phenomenon in urinary tract infection.
\end{abstract}

\section{Introduction}

Isolation of more than one bacterial species in a significant bacteriuria has been taken to indicate chronic or complicated infection [1]. Polymicrobial urosepsis carries a significantly higher mortality than monomicrobial urosepsis, particularly when complicated by bacteraemia [2], and the detection of true significant polymicrobial bacteriuria can serve as a non-invasive marker of underlying abnormalities [3]. Little attention has been given to the possibility of heterogeneity in single-species bacterial cultures from urinary tract infections. Colonial variation within a single strain of Escherichia coli can lead to an erroneous diagnosis of a mixed culture. This is a recognised problem [4], but generally it seems to be assumed that cultures are pure if they appear to be of a single species, are colonially homogeneous and the level of bacteriuria is significant. Therefore, the presence of more than one strain of the same species in a urine culture is likely to pass undetected in most routine laboratories.

In this study, the homogeneity of apparently pure cultures of bacteria from urine samples was investi-

Received 5 Sept. 1995; revised version received 15 Feb. 1996; accepted 6 March 1996.

Corresponding author: Dr R. Freeman. gated by pyrolysis mass spectrometry (PyMS), a highly discriminatory method for comparison of bacterial isolates [5].

\section{Materials and methods}

\section{Isolates}

Ten consecutive urine specimens yielding a significant $(>100000 \mathrm{cfu} / \mathrm{ml})$ and apparently pure culture of $E$. coli were studied. All the specimens were inoculated for culture within $2 \mathrm{~h}$ of being obtained from the patient. Brief clinical details are listed in Table 1. Each isolate was identified as $E$. coli by the API $20 \mathrm{E}$ system. Cultures exhibiting colonial variation were excluded [4]. The urine isolates were examined in five batches, each comprising isolates from two urine samples.

Ten distinct colonies randomly selected from the primary culture plate were subcultured twice on to two halves of a nutrient agar plate to give semiconfluent growth. These nutrient agar plates were taken from a single batch of medium. The subcultures were incubated overnight at $37^{\circ} \mathrm{C}$. As part of the initial diagnostic procedures each primary culture was tested for susceptibility to ampicillin, trimethoprim, cephalexin, gentamicin, cefuroxime and ciprofloxacin by a standard disk diffusion method and this was repeated on the single colony subcultures. 
Table 1. Details of urine specimens from which randomly selected colonies of $E$. coli were subcultured for examination by PyMS

\begin{tabular}{|c|c|c|c|c|c|c|c|c|c|c|}
\hline \multirow{2}{*}{$\begin{array}{l}\text { Urine } \\
\text { sample } \\
\text { no. }\end{array}$} & \multirow{2}{*}{$\begin{array}{l}\text { Specimen } \\
\text { type }\end{array}$} & \multicolumn{3}{|c|}{ Clinical details } & \multicolumn{6}{|c|}{ Antibiogram of primary isolate } \\
\hline & & Age (years) & Sex & Department & A & $\mathrm{T}$ & Cep & $\mathrm{G}$ & Cef & Cip \\
\hline 1 & $\mathrm{CSU}$ & 45 & $\mathrm{~F}$ & Urology & $\mathrm{r}$ & $\mathrm{s}$ & $\mathrm{s}$ & $\mathrm{s}$ & s & $\mathrm{s}$ \\
\hline 2 & $\mathrm{CSU}$ & 59 & M & Urology & $r$ & $\mathrm{r}$ & s & $\mathrm{s}$ & $\mathrm{s}$ & $\mathrm{s}$ \\
\hline 3 & MSU & 37 & $\mathbf{F}$ & Outpatient & $\mathrm{s}$ & $\mathrm{s}$ & s & $\mathrm{s}$ & s & $\mathrm{s}$ \\
\hline 4 & MSU & 30 & $\mathrm{~F}$ & General practice & $\mathrm{s}$ & $\mathrm{s}$ & $\mathrm{s}$ & $\mathrm{s}$ & $\mathrm{s}$ & $\mathrm{s}$ \\
\hline 5 & MSU & 45 & $\mathrm{M}$ & Cardiac surgery & $\mathrm{s}$ & $\mathrm{s}$ & $\mathrm{s}$ & $\mathrm{s}$ & $\mathrm{s}$ & $\mathrm{s}$ \\
\hline 6 & MSU & 45 & $\mathrm{M}$ & Patient 5 (repeat) & $\mathbf{s}$ & $\mathrm{s}$ & s & $\mathrm{s}$ & $\mathrm{s}$ & $\mathrm{s}$ \\
\hline 7 & MSU & 57 & $\mathrm{M}$ & Urology & $\mathrm{s}$ & $\mathrm{s}$ & $\mathrm{s}$ & $\mathrm{s}$ & $\mathrm{s}$ & $\mathrm{s}$ \\
\hline 8 & MSU & 34 & $\mathrm{~F}$ & General practice & $r$ & $r$ & $\mathrm{~s}$ & $\mathrm{~s}$ & $\mathrm{~s}$ & $\mathrm{~s}$ \\
\hline 9 & MSU & 45 & $\mathrm{M}$ & Nephrology & $\mathrm{s}$ & $\mathrm{s}$ & $\mathrm{s}$ & $\mathrm{s}$ & s & $\mathrm{s}$ \\
\hline 10 & MSU & 57 & $\mathrm{M}$ & General medicine & $\mathrm{r}$ & $\mathrm{r}$ & $\mathrm{s}$ & $\mathbf{s}$ & $\mathrm{s}$ & S \\
\hline
\end{tabular}

MSU, midstream urine; CSU, catheter urine; A, ampicillin; T, trimethoprim; Cep, cephalexin; G, gentamicin; Cef, cefuroxime; Cip, ciprofloxacin; r, resistant; s, susceptible.

\section{Pyrolysis mass spectrometry}

Each batch was examined separately but in the same manner, as described previously [6]. Colony material from each duplicate subculture of each isolate was smeared on to three pyrolysis foils (Horizon Instruments Ltd, Heathfield, Sussex) and the foils were heated at $80^{\circ} \mathrm{C}$ for $5 \mathrm{~min}$. All samples in the batch were processed on a Horizon Instruments PyMS 200X pyrolysis mass spectrometer, pyrolysing at $530^{\circ} \mathrm{C}$ for $4 \mathrm{~s}$.

Integrated ion counts at unit mass intervals of $51-200$ were recorded on to floppy disk, together with the pyrolysis sequence number and the total ion count for each sample. Spectra from the triplicate samples of each of the duplicate subcultures of each isolate were regarded as six-fold replicate analyses.

\section{Data analysis}

This has been described previously [7]. Briefly, after initial correction for variation in sample size (normalisation) the data were submitted to principal component $(\mathrm{PC})$ and canonical variate $(\mathrm{CV})$ analysis. Results were represented as an ordination diagram of PCCV1 versus PCCV2. Each point on the ordination diagram represented the mean spectrum for one of the original colonies, encircled by the $95 \%$ confidence limit boundary. The ordination diagram was inspected for outlying points, which were accepted as distinct if the $95 \%$ confidence limits did not overlap or abut those for other isolates. Data for such isolates were removed and the PCCV analysis was repeated. The endpoint of the analysis was reached when the $95 \%$ confidence limits overlapped for the remaining isolates and these were regarded as indistinguishable by PyMS.

\section{Results}

Isolates from urine sample 1 were clearly distinct from those of urine sample 2 (Fig. 1a). In further analysis, all isolates from urine sample 1 were indistinguishable. However, three isolates $(2 c, 2 g$ and $2 \mathrm{i}$ ) from urine sample 2 were clearly distinct from one another, and from the remaining seven isolates from sample 2, which formed a compact indistinguishable cluster (Fig. 1b).

Isolates from urine samples 3 and 4 formed two distinct clusters, with no evidence of heterogeneity within either specimen (Fig. 1c). In batch 3 one isolate (5f) was distinct (Fig. 1d) and the remaining 19 isolates from these sequential specimens from the same patient proved indistinguishable on further analysis.

In batch 4 , isolates from urines samples 7 and 8 again formed two compact distinct clusters, with one distinct isolate $(8 \mathrm{~g})$. No further heterogeneity was detected within isolates from either specimen (Fig. 1e). In batch 5 , all isolates from urine sample 10 proved indistinguishable, but isolates from urine sample 9 were heterogeneous, forming two distinct homogeneous clusters, one comprising isolates $9 \mathrm{c}, 9 \mathrm{f}$ and $9 \mathrm{j}$ and the other the remaining colonies from sample 9 (Fig. 1f).

There were no discrepancies between the antibiograms

Fig. 1. Ordination diagrams of the first versus the second principal component canonical variate function (PCCV1 versus PCCV2) from the analysis of spectral data for isolates from urines samples 1 and 2 (a), 10 colonies from urine sample 2 (b), the isolates from samples 3 and 4 (c), the isolates from samples 5 and 6 (d), the isolates from samples 7 and 8 (e) and from the isolates from samples 9 and 10 (f). In each case, individual points represent the mean spectral data from one colony (six foils) and the circles indicate $95 \%$ confidence limits. The overall outline of the cluster is shown where confidence limits overlap. 

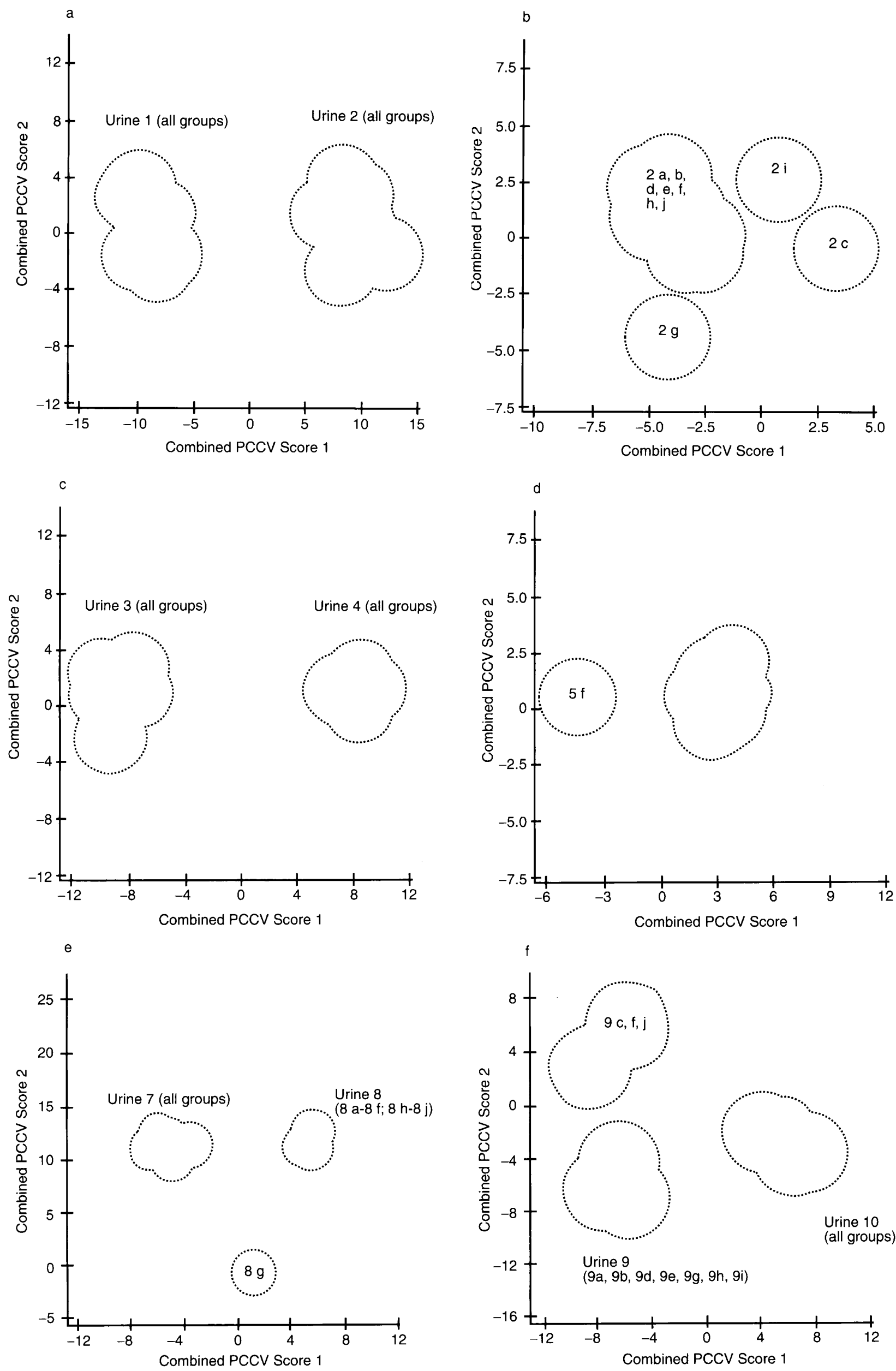
of the primary cultures and those of the appropriately related subcultured colonies.

\section{Discussion}

The results show clear heterogeneity in four of the 10 apparently pure cultures selected. Analysis of batches 1, 2, 4 and 5 showed that PyMS distinguished clearly between cultures from different patients and, therefore, presumably different strains of $E$. coli. Conversely, the results from batch 3 showed that the analysis did not falsely distinguish between two sequential cultures of the same strain.

The differences found between colonies from the same urine sample were of the same order as those found between putatively different strains from different patients. There was no evidence in this study that the differences were related to antibiograms. However, relatively small phenotypic variations can produce quite large differences in PyMS analyses [8]; therefore, it is important to extend the present study with genotyping. Furthermore, this study is one of the few instances in which primary cultures have been examined in PyMS studies. Most PyMS studies have been on collections of isolates which have undergone serial subcultures before examination. It is possible that the heterogeneity seen here is a more general phenomenon, reflecting a phenotypic diversity characteristic of all fresh isolates, itself worthy of further investigation.

Whatever the basis of the heterogeneity proves to be, there are at least two consequences for the study of urinary tract infections. Firstly, any study of the properties of uropathogenic strains of $E$. coli must embrace the possibility of variable but significant heterogeneity between colonies in an apparently pure recent culture. Conclusions based on the examination of single colonies or of 'sweeps' may need to be reassessed. Secondly, it is important to assess the clinical significance of colonial heterogeneity in fresh urine cultures. Should it emerge that heterogeneous cultures are more (or less) likely in chronic or complicated urinary tract infection, the observation might be the basis of a non-invasive test.

\section{References}

1. Kass EH. Symposium on new aspects of antibiotics: chemotherapeutic and antibiotic drugs in the management of infections of the urinary tract. Am $J$ Med 1955; 18: 764-781.

2. Siegman-Igra Y, Kulka T, Schwartz D, Konforti N. Polymicrobial and monomicrobial bacteraemic urinary tract infection. $J$ Hosp Infect 1994; 28: 49-56.

3. Gross PA, Flower M, Barden G. Polymicrobic bacteriuria: significant association with bacteremia. J Clin Microbiol 1976; 3: $246-250$.

4. Nichols GL. Variants of Escherichia coli giving the appearance of mixed growths in urine. J Clin Pathol 1975; 28: 728-730.

5. Freeman R, Goodfellow M, Gould FK, Hudson SJ, Lightfoot NF. Pyrolysis-mass spectrometry (Py-MS) for the rapid epidemiological typing of clinically significant bacterial pathogens. $J$ Med Microbiol 1990; 32: 283-286.

6. White GC, Sisson PR, Freeman R, Cookson BD. Rapid characterisation of Candida albicans by pyrolysis mass spectrometry. J Med Microbiol 1994; 41: 98-105.

7. Corkill JE, Sisson PR, Smyth A et al. Application of pyrolysis mass spectrometry and SDS-PAGE in the study of the epidemiology of Pseudomonas cepacia in cystic fibrosis. J Med Microbiol 1994; 41: 106-111.

8. Voorhees KJ, Durfee SL, Updegraff DM. Identification of diverse bacteria grown under diverse conditions using pyrolysis mass spectrometry. J Microbiol Methods 1988; 8: 315-325. 